\title{
Assessment of Tp-e interval, Tp-e/QT, and Tp-e/QTc ratios, in patients with COVID-19 infected with or without pneumonia
}

\author{
(DŞ̧aban Keleşoğlu' ${ }^{1}$, @Yücel Yılmaz ${ }^{2}$ \\ ${ }^{1}$ Erciyes University Medicine Faculty, Department of Cardiology, Kayseri, Turkey \\ ${ }^{2}$ Kayseri City Hospital, Kayseri, Turkey
}

Cite this article as: Keleşoğlu Ş, Yılmaz Y. Assessment of Tp-e interval, Tp-e/QT, and Tp-e/QTc ratios, in patients with COVID-19 infected with or without pneumonia. J Health Sci Med 2021; 4(5): 615-621.

\begin{abstract}
Objective: COVID-19 infection has the potential to affect the cardiovascular system as well as respiratory disease. Recent studies have suggested that Tp-e interval, Tp-e/QT, and Tp-e/QTc ratios may be associated with ventricular arrhythmias. The goal of this study is to the evaluation of Tp-e interval, Tp-e/QT, and Tp-e/QTc ratios, in patients with COVID-19 infected with or without pneumonia.

Material and Method: 118 patients with COVID-19 infection were divided into 2 groups: patients with and without pneumonia, the control group consisted of 39 people. The Tp-e interval, Tp-e/QT, and Tp-e/QTc ratio were gauged by the 12 lead electrocardiogram.

Results: Tp-e interval, Tp-e/QT, Tp-e/QTc ratio were considerably high in COVID-19 patients with pneumonia, contrasted to the without pneumonia patients. ( $<<0.01, \mathrm{p}<0.01, \mathrm{p}<0,01$, respectively) whereas the same parameters were similar to the control group in COVID-19 infected patients without pneumonia. ( $\mathrm{p}=0.258, \mathrm{p}=0.249, \mathrm{p}=0,056$, respectively). Correlation analysis revealed a significant and positive correlation between CRP with Tp-e interval, Tp-e/QT ratio, and Tp-e/QTc ratio. There was the same correlation relationship between WBC with Tp-e interval, Tp-e/QT ratio, and Tp-e/QTc ratio.

Conclusion: Our present study showed that Tp-e interval, Tp-e/QT, Tp-e/QTc ratio were prolonged in COVID-19 infected patients with pneumonia.
\end{abstract}

Keywords: COVID-19, Tp-e interval, Tp-e/QT and Tp-e/QTc

\section{INTRODUCTION}

Coronavirus disease 2019 (COVID-19), which originated in China in late December 2019 and was effective all over the world in a very short time, was declared as a pandemic by the World Health Organization.

COVID-19 named severe acute respiratory syndrome coronavirus 2 (SARS-CoV-2), is a novel RNA virus that induces acute pneumonia that can lead to severe respiratory distress syndrome, which may be fatal. Nonetheless, many infections are mild or asymptomatic $(1,2)$.

Although COVID-19 primarily affects the lungs, it can also affect multiple organs, especially the cardiovascular system. Acute cardiovascular events complicating the clinical course of COVID-19 may be one of the causes of poor survival. One of the most common cardiac complications during this disease is arrhythmias $(3,4)$. It has been shown that the arrhythmic event was observed between $7 \%$ to $44 \%$ depending on the severity of the disease (5). Arrhythmias such as atrial fibrillation, conduction blocks, ventricular tachycardia, and ventricular fibrillation have been shown to occur in the course of COVID-19 $(3,4)$.

In ECG, research of ventricular recovery and augmented dispersion of repolarization are useful markers for ventricular arrhythmias. Some ventricular repolarization markers are useful to predict arrhythmias, including the QT interval, QT dispersion, and T-wave alternans $(6,7)$.

Recent studies have suggested that new indexes such as Tpeak-Tend (Tp-e) interval, and Tp-e interval/QT interval (Tp-e/QT) ratio may be associated with ventricular arrhythmias in various clinical scenarios $(8,9)$.

We purposed to examine the changes in Tp-e interval, $\mathrm{Tp}-\mathrm{e} / \mathrm{QT}$ ratio and Tp-e/QTc ratio in adults infected COVID-19 patients with and without pneumonia. 


\section{MATERIAL AND METHOD}

Ethical approval for the study was granted by Kayseri City Hospital Clinical Research Ethics Committee (Date: 25.06.2020, Decision No: 135). All procedures were carried out in accordance with the ethical rules and the principles of the Declaration of Helsinki.

\section{Study population}

Our study was designed as a single-center study regarding the confirmed COVID-19 patients monitored and treated in inpatient clinics, or the intensive care units (ICUs).

The study was carried out in our hospital, which accepted COVID-19 patients who were confirmed by molecular methods determined by the Ministry of Health of Turkey as ' COVID-19 Hospitals'. Electrocardiographic data of the patients were recorded, including the risk factors, as well as clinical, radiological, and laboratory findings.

Patients older than 18 years of age and were diagnosed as definite SARS-CoV2 in presence of symptoms and positive polymerase chain reaction (PCR) were analyzed. In this cross-sectional study, a total of 118 patients hospitalized with COVID-19 diagnosis between March and June 2020 in our hospital were evaluated. 39 well- sex and age-matched patients with randomized selected for the comparison were included in the study.

Patients were separated into two groups according to the clinical presentation based on the definitions in the "COVID-19 Diagnosis and Treatment Guide" printed by the Turkish Ministry of Health (10).

Group 1 Mild illness presents with features such as fever, muscle/joint pain, cough, sore throat, and nasal congestion, without pneumonia together with a respiratory rate $<30 / \mathrm{min}$ and an $\mathrm{O} 2$ saturation above $90 \%$ while breathing room air.

Group 2 Severe illness is defined with widespread findings of pneumonia in chest radiography or computed tomography.

The first electrocardiogram (ECG) recording of patients at the time of hospital admission were analyzed. At that time, no patient was receiving any medical treatment. Patients with a history of ischemic heart disease, those with a history of heart valve disease, those with heart failure (left ventricular ejection fraction $\leq 50$ ), those with a branch block or atrioventricular conduction disorder in the surface ECG, those with poor electrolyte imbalance, patients with poor ECG quality were excluded from the study.

The standard COVID-19 treatment protocol recommendedbytheScienceAdvisoryBoard of theTurkish Ministry of Health, including, hydroxychloroquine 200 $\mathrm{mg}$ twice daily, and azithromycin $250 \mathrm{mg}$ once daily following a $500 \mathrm{mg}$ loading dose were administered to all patients. In addition to patients with pneumonia, Oseltamivir phosphate $75 \mathrm{mg}$ was added twice Daily (10). Demographic data, findings of the imaging studies, and laboratory test results were retrieved from the institutional digital database.

If the serum levels of troponin I, one of the cardiac biomarkers, were below the upper reference limit of the 99th percentile or if there were no new abnormalities in electrocardiography and echocardiography, it was considered that there was no myocardial damage (11). Hypertension, Diabetes mellitus, and Hyperlipidemia defined as previously described (12). Smokers were current smokers and had been using for at least 10 years.

\section{Electrocardiogram (ECG) Analysis}

All standard 12-lead ECGs were acquired at rest in the supine position simultaneously using a recorder (Philips brand machine) set at $25 \mathrm{~mm} / \mathrm{s}$ paper speed and $1 \mathrm{mV} / \mathrm{cm}$ standardization. All ECGs were scanned and transferred to personal computers an electronic caliper (Cardio Calipers, version 3.3 software; Iconico.com, Philadelphia, PA, USA) was used under magnification to record the measurements. Assessments of the ECG were done by 2 cardiologists blinded to the clinical data, and to diminish the error measurements. Patients whose ECGs showed $\mathrm{U}$ waves and negative $\mathrm{T}$ waves were excluded from the study.

The Tp-e interval was defined as the distance between the peak of the T-wave and the end of the T-wave. All Tp-e intervals were measured using the best available $\mathrm{T}$-wave in lead V5 (13). When the lead V5 result was not suitable for analysis, the V4 and V6 were used.

The QT interval was measured from the beginning of the QRS complex to the end of the $\mathrm{T}$ wave in precordial lead V6, which best reflects the transmural axis of the left ventricle (14) and corrected for HR using the Bazett formula: $\mathrm{cQT}=\mathrm{QT} \sqrt{ }(\mathrm{R}-\mathrm{R}$ interval. The $\mathrm{Tp}-\mathrm{e} / \mathrm{QT}$ ratio and Tp-e/QTc (Tp-e divided by QT and Tp-e divided by $\mathrm{QTc}$ ) were calculated from these measurements. Interobserver and intraobserver coefficients of variation were less than $5 \%$, respectively.

\section{Echocardiography}

Conventional echocardiography was performed with Philips Epiq 7 ultrasound system (Philips, Andover, Mass., USA). To reduce the risk of COVID-19 transmission, only LVEF and left ventricular dimensions were examined to detect myocardial damage. Conventional echocardiographic images were obtained from the parasternal and apical views The teichholz method was used for the calculation of LV 
ejection fraction.

\section{Statistical Analysis}

Statistical analyzes were performed using SPSS Statistics Package version 21.0 (SPSS Inc, Chicago, IL, USA) for Windows. The normal distribution of variables was analyzed using the Kolmogorov-Smirnov method. Continuous data mean and standard deviations were evaluated and recorded. Continuous variable distribution among the groups was done by One Way Anova. Variability between groups was performed by the LSD test. The chisquare test was used for categorical variables and was calculated as a percentage. The relationship between the variables was analyzed by Pearson correlation analysis. P-value less than 0.05 was considered significant.

\section{RESULTS}

A total of 157 participants were selected in the current study. The COVID-19 group consisted of 118 subjects (64 men, 54 female), and the control group included 39 individuals ( 22 men, 17 female). COVID-19 Patients were divided into two groups according to the presence of pneumonia. While 48 of these patients had no pneumonia (group 1), 70 had pneumonia (group 2).

Baseline clinical and demographic features of the study groups are presented in Table 1. The study population was similar regarding sex distribution, age, smoking status, frequencies of hypertension, and diabetes were not significantly different between patients and the control group ( $\mathrm{p}>0.05)$.

The baseline laboratory measurements of the study patients are listed in Table 2. Serum glucose, C-reactive protein (CRP), white blood cell (WBC), and platelet levels were significantly higher COVID -19 with pneumonia patients. ( $\mathrm{p}<0,01, \mathrm{p}<0,01, \mathrm{p}<0,01, \mathrm{p}<0,01$ respectively). Other blood parameters were similar between groups. Troponin values in COVID patients with and without pneumonia were within the normal range. There was no difference between the groups.

The electrocardiographic and echocardiography parameters of the groups are shown in Table 3. LVEF, an indicator of LV systolic function, was similar between groups ( $\mathrm{p}=0.432)$. The $\mathrm{QRS}$ duration, $\mathrm{QT}$ interval, and QTc interval were similar between the groups ( $p=0,054$, $\mathrm{p}=0.858, \mathrm{p}=0,221$ respectively) Although heart rate in

\begin{tabular}{|c|c|c|c|c|}
\hline Variables & $\begin{array}{c}\text { Control } \\
\text { group } \\
(\mathrm{n}=39)\end{array}$ & $\begin{array}{c}\text { Covid+ without } \\
\text { pneumonia } \\
(\mathrm{n}=48)\end{array}$ & $\begin{array}{c}\text { Covid+ with } \\
\text { pneumonia } \\
(n=70)\end{array}$ & $\begin{array}{c}\text { p } \\
\text { value }\end{array}$ \\
\hline Age (years) & $49 \pm 10.716$ & $49.81 \pm 12.489$ & $52.91 \pm 16.182$ & 0.186 \\
\hline Male/female & 22/17 & $25 / 23$ & $39 / 31$ & 0.901 \\
\hline HT & 5 & 6 & 18 & 0.111 \\
\hline DM & 2 & 2 & 9 & 0.173 \\
\hline Smoking & 7 & 9 & 13 & 0.995 \\
\hline
\end{tabular}

\begin{tabular}{|c|c|c|c|c|}
\hline Variables & Control group (39) & Covid+ without pneumonia (48) & Covid+ with pneumonia (70) & P value \\
\hline Glucose (mg/dL) & $86.41 \pm 8.303$ & $98.42 \pm 26.026$ & $107.23 \pm 25.043$ & $<0.01^{\star}$ \\
\hline Kreatinin (mg/dL) & $0.849 \pm 0.176$ & $0.804 \pm 0.186$ & $0.888 \pm 0.288$ & 0.162 \\
\hline AST (U/L) & $22.36 \pm 6.247$ & $20.65 \pm 7.292$ & $23.86 \pm 12.308$ & 0.211 \\
\hline $\operatorname{ALT}(\mathrm{U} / \mathrm{L})$ & $19.26 \pm 7.312$ & $20.65 \pm 12.80$ & $22.47 \pm 14.778$ & 0.429 \\
\hline Total Bilirubin (mg/dL) & $0.58 \pm 0.207$ & $0.52 \pm 0.350$ & $0.57 \pm 0.319$ & 0.607 \\
\hline CRP & $2.78 \pm 1.077$ & $9.708 \pm 11.33$ & $80.934 \pm 12.703$ & $<0.01^{\star}$ \\
\hline WBC $\left(10^{\wedge} 3 / \mathrm{uL}\right)$ & $8.428 \pm 2.503$ & $7.506 \pm 2.420$ & $17.276 \pm 4.420$ & $<0.01^{\star}$ \\
\hline Hemoglobin $(\mathrm{g} / \mathrm{l})$ & $13.682 \pm 1.738$ & $13.913 \pm 1.9513$ & $14.013 \pm 2.3727$ & 0.115 \\
\hline Platelet (/mm3) & $233.74 \pm 81.398$ & $236.69 \pm 55.237$ & $278.51 \pm 83.285$ & $0.002^{*}$ \\
\hline Troponin I & & $<0.1$ & $<0.1$ & 1.000 \\
\hline
\end{tabular}

Table 3. Electrocardiographic characteristics of the study population

\begin{tabular}{|c|c|c|c|c|}
\hline Variables & Control group (39) & Covid+ without pneumonia (48) & Covid+ with pneumonia (70) & $\mathbf{p}$ \\
\hline Heart rate (beat/min) & $75.90 \pm 8.641$ & $74.71 \pm 11.187$ & $70.21 \pm 9.824$ & $0.007^{\star}$ \\
\hline QRS duration (ms) & $84.72 \pm 8.392$ & $98.96 \pm 28.896$ & $84.89 \pm 10.001$ & 0.054 \\
\hline QT interval (ms) & $380.46 \pm 20.963$ & $378.42 \pm 27.546$ & $381.12 \pm 27.782$ & 0.858 \\
\hline QTc interval (ms) & $422.77 \pm 16.539$ & $417.44 \pm 14.599$ & $416.56 \pm 14.478$ & 0.221 \\
\hline Tp-e (ms) & $74.08 \pm 4.922$ & $75.13 \pm 5.511$ & $84.97 \pm 4.380$ & $<0.01^{\star}$ \\
\hline TPe/QTc ratio (ms) & $0.175 \pm 0.011$ & $0.179 \pm 0.11$ & $0.209 \pm 0.010$ & $<0.01^{*}$ \\
\hline TPe/QT ratio (ms) & $0.195 \pm 0.015$ & $0.199 \pm 0.019$ & $0.223 \pm 0.017$ & $<0.01^{*}$ \\
\hline $\operatorname{LVEF}(\%)$ & $65.05 \pm 4.57$ & $64.25 \pm 4.31$ & $63.97 \pm 3.88$ & 0.432 \\
\hline
\end{tabular}

Data are expressed as mean \pm standard deviation for normally distributed data and percentage $(\%)$ for categorical variables. Tp-e $=\mathrm{T}$ wave interval from peak to end, $\mathrm{c}=\mathrm{corrected}, \mathrm{P}$ values that are lower than 0.05 are marked with * 
the ECG was within the normal range, COVID-19 with pneumonia was significantly lower.

$\mathrm{Tp}$-e interval, Tp-e/QT ratio, and Tp-e/QTc ratio were significantly higher in COVID-infected with pneumonia patients compared to the COVID infected without pneumonia. $(\mathrm{p}<0.01)$ (Figure 1). Tp-e interval, Tp-e/ QT ratio, and Tp-e/QTc ratio were similar between COVID infected without pneumonia and control groups. $(\mathrm{p}=0,321, \mathrm{p}=0,56, \mathrm{p}=0,249)$ (Figure 1)

In correlation analysis revealed a significant and positive correlation between CRP with Tp-e interval, Tp-e/QT ratio, and Tp-e/QTc ratio ( $\mathrm{r}=0471, \mathrm{p}=0,001 ; \mathrm{r}=0,416$, $\mathrm{p}=0,001 ; \mathrm{r}=0,481, \mathrm{p}=0,001$ respectively) (Figure 2 ).

There was the same correlation relationship between WBC with Tp-e interval, Tp-e/QT ratio, and Tp-e/ QTc ratio. ( $r=550, p=0,001 ; \mathrm{r}=0,478, \mathrm{p}=0,001 ; \mathrm{r}=0,602$, $\mathrm{p}=0,001$ respectively).
Thirty-two of the 70 patients with COVID pneumonia required intensive care, although cardiac death was observed in 3 patients, but documented ventricular arrhythmia was not detected in these patients. However, both of these patients had prolonged T-pe (96 msn,93 msn,91 msn respectively), and T-pe/QTc ratios ( 0.22 , $0.21,0.21$ respectively).

\section{DISCUSSION}

The most important result of our work is that Tp-e interval, Tp-e/QT, and Tp-e/QTc ratios were prolonged in COVID-19 patients with pneumonia as compared to COVID-19 patients with mild disease without pneumonia. However, the results of COVID-19 patients with mild disease without pneumonia were similar to healthy ones. These findings may have an important role in the diagnosis and management of COVID-19 -related arrhythmias.

\section{Table 4. Post hoc analysis}

\begin{tabular}{|c|c|c|c|c|c|c|c|c|c|}
\hline & $\begin{array}{c}\text { Control } \\
\text { group }\end{array}$ & Group 1 & $\mathbf{p}$ & $\begin{array}{c}\text { Control } \\
\text { group }\end{array}$ & Group 2 & $\mathbf{p}$ & Group 1 & Group 2 & $\mathbf{p}$ \\
\hline Heart rate (beat/min) & 75.90 & 74.71 & 0.582 & 75.90 & 70.21 & $.005^{\star}$ & 74.71 & 70.21 & 0.018 \\
\hline QT (msn) & 380.46 & 378.42 & 0.718 & 380.46 & 381.12 & .903 & 378.42 & 381.12 & 0.585 \\
\hline QTc (msn) & 422.77 & 417.44 & 0.102 & 422.77 & 416.56 & .184 & 417.44 & 416.56 & 0.151 \\
\hline Tpe (msn) & 74.08 & 75.13 & 0.258 & 74.08 & 84.97 & $<0.01$ & 75.13 & 84.97 & $<0.01$ \\
\hline TPe/QTc ratio (ms) & 0.175 & 0.179 & 0.056 & 0.175 & 0.209 & $<0.01$ & 0.179 & 0.209 & $<0.01$ \\
\hline $\mathrm{WBC}$ & 8.42 & 7.50 & 0.229 & 8.42 & 17.27 & $<0.01$ & 7.50 & 17.27 & $<0.01$ \\
\hline CRP & 2.78 & 9.70 & $<0.01$ & 2.78 & 80.93 & $<0.01$ & 9.70 & 80.93 & $<0.01$ \\
\hline
\end{tabular}

Data are expressed as mean \pm standard deviation for normally distributed data and percentage (\%) for categorical variables. Group1. Covid + without pneumonia, Group 2 . Covid+with pneumonia

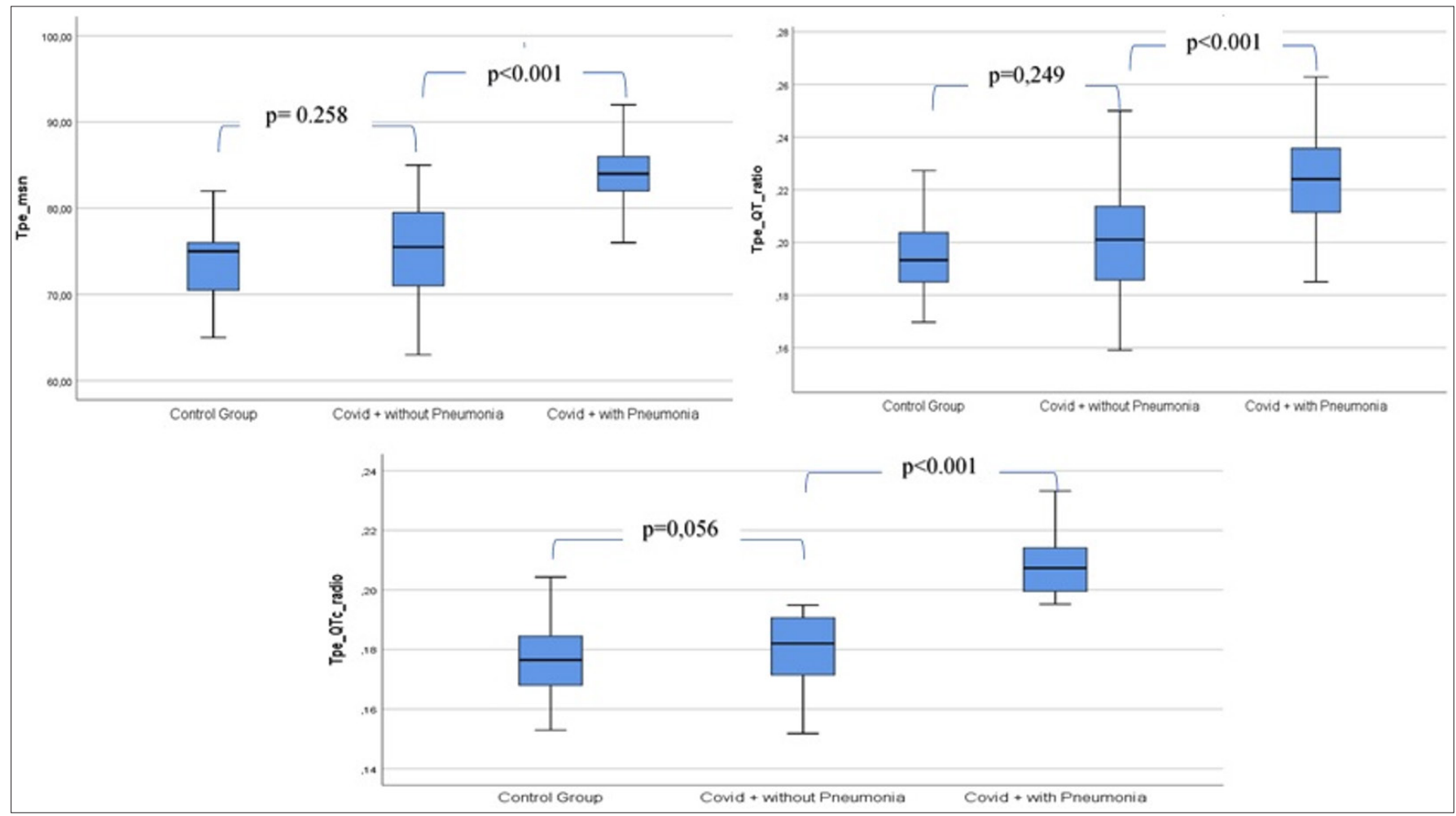

Figure 1. Change of Tp-e, Tp-e/QT ratio, and Tp-e/QTc ratio between study groups. 


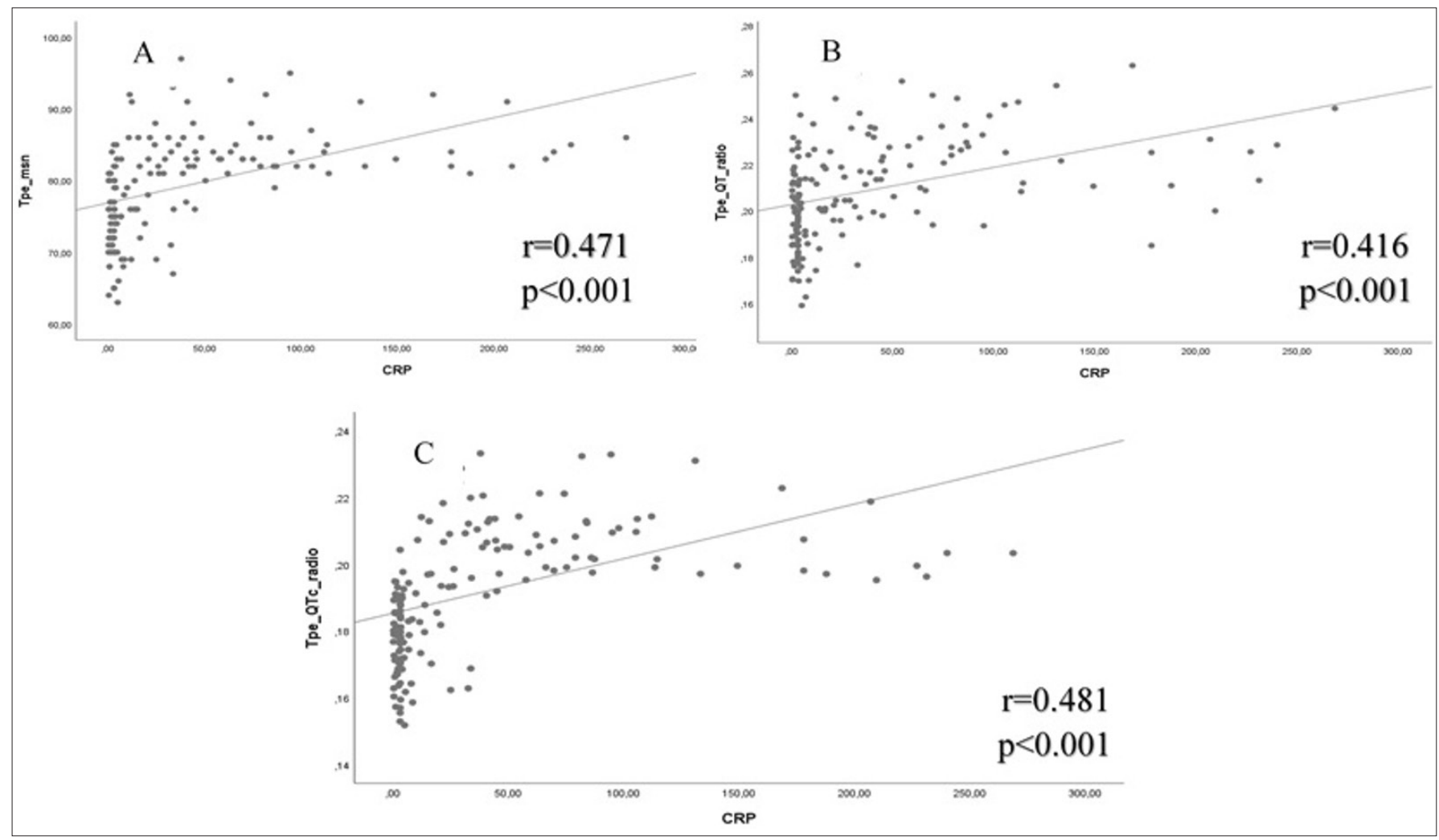

Figure 2. (A) Correlation between Tp-e interval and CRP count. (B) Correlation between Tp-e/QT ratio and CRP count. (C) Correlation between Tp-e/QTc ratio and CRP count

Shi et al. (15) from china reported that $16.7 \%$ of hospitalized COVID-19 patients and 44.4\% of COVID-19 intensive care patients had malignant arrhythmias. Some 12-lead ECG parameters as potential markers of malignant arrhythmias have been proposed.

In a previous study, the QTc interval and QT dispersion which show myocardial repolarization status have been used for risk stratification in different patient groups (16). In recent years, the use of Tp-e interval and Tp-e/QTc ratio in determining ventricular arrhythmias and risk of sudden cardiac death has become increasingly common. Previous studies have claimed that the mechanism of association of prolonged QT interval, Tp-e interval, and sudden cardiac deaths is a tendency to ventricular reentry which causes ventricular arrhythmias (17).

Because Tp-e/QTc ratio is not affected by alteration in heart rate and interpersonal variation of QT interval, the $\mathrm{Tp}-\mathrm{e} / \mathrm{QTc}$ ratio is a more useful parameter than Tp-e and QT intervals (13).

In our study found out that Tp-e interval, Tp-e/QT, and Tp-e/QTc ratios which are the most susceptible index of myocardial repolarization and which may show a higher tendency to ventricular arrhythmia and sudden cardiac death were meaningfully prolonged in COVID-19 patients with pneumonia. The same values are similar to healthy people in those who have mild disease despite having COVID-19. that is, we found that the probability of ventricular arrhythmia does not increase in this patient group. Therefore, the risk of ventricular arrhythmia in these individuals can be said to be similar to that of the healthy.

Hence, these results imply that the risk of development of arrhythmias is higher in COVID-19 infected patients with pneumonia. However, this status only was seen in patients who had experience pneumonia. What could the possible mechanisms behind the relationship we observed with COVID-19 infection in this study?

Although studies about cardiac damage and long-term effects of COVID-19 are very recent and uncertain, there is remaining, evidence that it can induce cardiac pathologies and/or exacerbate existing cardiovascular diseases. COVID-19, possible mechanisms involved in the physiopathology of cardiac injury are as follows: Direct cardiotoxic effect (myocarditis) through ACE2 receptor which plays an essential role in the pathogenesis of the virus, increased sympathetic stimulation, hypoxia due to lung involvement, productions of pro-inflammatory cytokines, such as IL- $1 \beta$, IL- 6 , and TNF- $\alpha$ secondary to inflammation and increased clotting tendency could be the possible mechanisms (18).

Myocarditis can be seen in the course of the disease, although it is very rare (19). Huang et al. (20) demonstrated that COVID-19 linked with myocardial damage occurred in approximately $12.1 \%$ of patients and appeared as an increase in hs-TnI levels.

In a study by Ucar et al. (21) in patients with myocarditis, 
they reported that there is a prolongation of the Tp-e interval and $\mathrm{Tp}-\mathrm{e} / \mathrm{QT}$ ratio due to local inflammation and fibrosis of the myocardium.

In our study, both because LVEF was normal in echocardiography and blood troponin I levels was normal has removed us from the diagnosis of possible myocarditis. For this reason, the change in the Tp-e interval, Tp-e/QT, and Tp-e/QTc ratios index in our study is considered to be independent of myocarditis.

The mechanism of cardiac and arrhythmic complications may be associated with pro-inflammatory mediators which increase COVID-19 infection. Production of proinflammatory cytokines secondary to inflammation, such as IL-1 $\beta$, IL-6, IL-18, and TNF-a, has been shown to increase in patients with severe COVID-19 infections by Madjid et al. (22).

In previous studies on transgenic mouse models, a strong relationship has been demonstrated between inflammation and pro-inflammatory cytokines (TNFa and IL-1 $\beta$ ) and arrhythmia formation (23). Lagrand et al. (24) showed that CRP can cause direct arrhythmias by activating complement and inducing oxidative stress and apoptosis. CRP level which conventionally uses in infectious diseases is correlated with several cytokine levels such as interleukin (IL)-6 and tumor necrosis factoralpha (TNFa) levels (25). As known, local or circulating pro-inflammatory cytokines are correlated with plasma CRP levels. The greater the severity of the inflammatory stimulus, the greater the change in the concentrations of the acute phase proteins such as CRP. That is, CRP correlates with the extent and severity of the disease. Therefore, in our study a markedly increase in CRP levels in COVID-19 patients who have had pneumonia, suggests that these patients had a stronger inflammatory response. In light of these data, it can be concluded that the increase in CRP increases the incidence of arrhythmia. It can be said that these patients are more sensitive to ventricular arrhythmia because the inflammation increases the risk of arrhythmia in COVID-19 pneumonia patients. Moreover, this study demonstrated that the inflammatory marker CRP correlated with the Tp-e interval, Tp-e/QT ratio, and Tp-e/QTc ratios, positively. That's why it can be said that the risk of arrhythmia increases significantly due to the increase in the CRP level. Therefore, we can consider that this present study's results are similar to the previous results.

In COVID-19 patients without pneumonia, it can be thought that the CRP levels are significantly lower, resulting in a much less inflammatory response. The similarity of the risk of ventricular arrhythmia to healthy people in these patients can be explained by an insufficient inflammatory response. In this study.
Soto-Gomez et al. (26) found an increase in newly started arrhythmic events in patients hospitalized for pneumonia. So, they claimed that pneumonia is an arrhythmogenic disease. It has been shown by Restrepo et al. (27) that pneumonia affects the cardiovascular system with multiple mechanisms and causes cardiac arrhythmias. Similar results have been shown by Corrales-Medina et al. (28). Possible causes, according to them, can be listed as follows: increase in serum inflammatory cytokines, acute physiologic or metabolic disorders related to pneumonia, such as hypo/hyperthermia, electrolyte abnormalities, and hypoxemia, may trigger arrhythmias. The development of pneumonia in COVID-19 infected patients may have increased the risk of arrhythmia by the mentioned mechanisms. Therefore, it is reasonable that Tp-e, Tpe/QTc ratio values, which are an indicator of possible ventricular arrhythmia, are significantly prolonged in patients with pneumonia, as in our study. Also, in our study, the most important finding that supports these data is the correlation finding between inflammation parameters and $\mathrm{Tp}-\mathrm{e} / \mathrm{QT}$ ratio and $\mathrm{Tp}-\mathrm{e} / \mathrm{QTc}$ ratios index.

Öztürk et al. (29) claimed that QTc, QTd, and Tp-e/QTc, were significantly prolonged in COVID-19 patients. However, in this study, the number of patients is limited, and the severity of the disease is uncertain. In our study, we divided COVID-19 patients into 2 groups according to the presence or absence of pneumonia, which we think indicates the severity and viral load of the disease. In our study, we found that arrhythmia potential was present only in COVID-19 patients who developed pneumonia rather than all COVID-19 patients. This is the most crucial data of our study.

\section{CONCLUSION}

When the results obtained in our study are evaluated; abnormal dispersion of ventricular repolarization has existed in severe COVID-19 patients. Considering that the Tp-e interval and Tp-e/QTc ratio have been used as the electrocardiographic index of ventricular arrhythmogenesis and sudden cardiac death in recent years, COVID-19 patients with more serious diseases such as pneumonia have a high risk for cardiac arrhythmia and sudden cardiac death. We think that patients with COVID pneumonia should be followed more closely for ventricular arrhythmia, especially since the drugs used in the treatment of COVID 19 have the potential to affect ventricular repolarization parameters. Also, ECG can be used to evaluate the risk of cardiac arrhythmia in severe COVID-19. We also think that this work is a pilot study for future research of the Tp-e interval, the Tp-e/QTc ratio, and COVID-19 related arrhythmia and mortality.

\section{Limitations}

The main limitations of our study are the relatively small 
number of patients in our study group to see if prolonged Tpe, Tpe/QTc ratio develop ventricular arrhythmias in COVID-19 patients and the lack of follow-up in terms of possible future ventricular arrhythmias.

\section{ETHICAL DECLARATIONS}

Ethics Committee Approval: Approval for the study was granted by Kayseri City Hospital Clinical Research Ethics Committee (Date: 25.06.2020, Decision No: 135).

Informed Consent: Because the study was designed retrospectively, no written informed consent form was obtained from patients.

Conflict of Interest Statement: The authors have no conflicts of interest to declare.

Financial Disclosure: The authors declared that this study has received no financial support.

Author Contributions: All of the authors declare that they have all participated in the design, execution, and analysis of the paper, and that they have approved the final version.

\section{REFERENCES}

1. Wu Z, McGoogan JM. Characteristics of and Important Lessons from the Coronavirus Disease 2019 (COVID-19) Outbreak in China: Summary of a Report of 72314 Cases from the Chinese Center for Disease Control and Prevention. JAMA 2020. doi: 10.1001/jama 2020. 2648.

2. Özdemir Ö. Coronavirus Disease 2019 (COVID-19): Diagnosis and Management. Erciyes Med J 2020; 42: 242-7.

3. Guzik TJ, Mohiddin SA, Dimarco A, et al. COVID-19 and the cardiovascular system: implications for risk assessment, diagnosis, and treatment options. Cardiovasc Res 2020; 116: 1666-87.

4. Kelesoglu S, Yilmaz Y, Ozkan E, et al. New onset atrial fibrilation and risk faktors in COVID-19. J Electrocardiol 2021; 65: 76-81.

5. Liu K, Fang YY, Deng Y, et al. Clinical characteristics of novel coronavirus cases in tertiary hospitals in Hubei Province. Chin Med J (Engl) 2020; 133: 1025-31.

6. Elming H, Holm E, Jun L, et al. The prognostic value of the QT interval and QT interval dispersion in all-cause and cardiac mortality and morbidity in a population of Danish citizens. Eur Heart J 1998; 19: 1391-400.

7. Tse G, Yan BP. Traditional and novel electrocardiographic conduction and repolarization markers of sudden cardiac death. Europace 2017; 19: 712-21.

8. Kors JA, Ritsema van Eck HJ, van Herpen G. The meaning of the Tp-Te interval and its diagnostic value. J Electrocardiol 2008; 41 575-80

9. Castro Hevia J, Antzelevitch C, Tornes Barzaga F, et al. Tpeak-T end and $\mathrm{T}$ peak- $\mathrm{T}$ end dispersion as risk factors for ventricular tachycardia/ventricular fibrillation in patients with the Brugada syndrome. J Am Coll Cardiol 2006; 47: 1828-34.

10.T.C. Ministry of Health General Directorate of Public Health, COVID-19 (Sars-Cov2 Infection) Directory, Coronavirus Scientific Advisory Board, Turkey 2020

11. Thygesen K, Alpert JS, Jaffe AS, et al. Fourth universal definition of myocardial infarction (2018). Eur Heart J 2019; 40: 237-69

12.Perk J, De Backer G, Gohlke H, et al. European Guidelines on cardiovascular disease prevention in clinical practice (version 2012). The Fifth Joint Task Force of the European Society of
Cardiology and Other Societies on Cardiovascular Disease Prevention in Clinical Practice (constituted by representatives of nine societies and by invited experts). Eur Heart J 2012; 33: 1635-701.

13. Gupta P, Patel C, Patel H, et al. Tp-e/QT ratio as an index of arrhythmogenesis. J Electrocardiol 2008; 41: 567-74.

14. Kors JA, van Herpen G, van Bemmel JH. QT dispersion as an attribute of T-loop morphology. Circulation 1999; 99: 1458-63.

15. Shi S, Qin M, Shen B, et al. Association of cardiac injury with mortality in hospitalized patients with COVID-19 in Wuhan, China. JAMA Cardiol 2020; 5: 13-364.

16. Barr CS, Naas A, Freeman M, Lang CC, Struthers AD. QT dispersion and sudden unexpected death in chronic heart failure. The Lancet 1994; 343: 327-9.

17. Antzelevitch C, Sicouri S, Di Diego JM, et al. Does Tpeak-Tend provide an index of transmural dispersion of repolarization? Heart Rhythm 2007; 4: 1114-6.

18. Tomasz J. Guzik et al. COVID-19 and the cardiovascular system: implications for risk assessment, diagnosis, and treatment options. Cardiovasc Res 2020.

19. Hu H, Ma F, Wei X, Fang Y. Coronavirus fulminant myocarditis saved with glucocorticoid and human immunoglobulin. Eur Heart J 2020.

20. Huang C, Wang Y, Li X, et al. Clinical features of patients infected with 2019 novel coronavirus in Wuhan, China. Lancet 2020; 395: 497-506

21.Ucar FM, Ozturk C, Yllmaztepe MA. Evaluation of Tp-e Interval, Tp-e/QT Ratio and Tp-e/QTc Ratio in Patients with Acute Myocarditis. BMC Cardiovasc Disord 2019; 19: 232

22. Madjid M, Safavi-Naeini P, Solomon SD, Vardeny O. Potential effects of coronaviruses on the cardiovascular system: a review. JAMA Cardiol 2020.

23. Brouillette J, Cyr S, Fiset C. Mechanisms of arrhythmia and sudden cardiac death in patients with HIV infection. Can J Cardiol 2019; 35: 310-9.

24.Lagrand WK, Visser CA, Hermens WT,et al. C-reactive protein as a cardiovascular risk factor: more than an epiphenomenon? Circulation 1999; 100: 96-102.

25. Kushner I. The phenomenon of the acute phase response. Ann New York Acad Sci 1982; 389: 39-48

26. Soto-Gomez N, Anzueto A, Waterer GW, Restrepo MI, Mortensen EM. Pneumonia: an arrhythmogenic disease? Am J Med 2013; 126: $43-8$.

27. Restrepo MI, Reyes LF. Pneumonia as a cardiovascular disease. Respirology 2018; 23: 250-9.

28. Corrales-Medina VF, Musher DM, Shachkina S, Chirinos JA. Acute pneumonia and the cardiovascular system. Lancet 2013; 381: 496-505

29. Öztürk F, Karaduman M, Çoldur R, İncecik Ş, Güneş Y, Tuncer M. Interpretation of arrhythmogenic effects of COVID-19 disease through ECG. Aging Male 2020: 1-4. 